\title{
Using Quantitative Analysis to Implement Autonomic IT Systems
}

\author{
Radu Calinescu and Marta Kwiatkowska \\ Computing Laboratory, University of Oxford \\ \{Radu.Calinescu, Marta.Kwiatkowska\}@ comlab.ox.ac.uk
}

\begin{abstract}
The software underpinning today's IT systems needs to adapt dynamically and predictably to rapid changes in system workload, environment and objectives. We describe a software framework that achieves such adaptiveness for IT systems whose components can be modelled as Markov chains. The framework comprises (i) an autonomic architecture that uses Markov-chain quantitative analysis to dynamically adjust the parameters of an IT system in line with its state, environment and objectives; and (ii) a method for developing instances of this architecture for real-world systems. Two case studies are presented that use the framework successfully for the dynamic power management of disk drives, and for the adaptive management of cluster availability within data centres, respectively.
\end{abstract}

\section{Introduction}

In order to support the latest advances in research, business and everyday services, software has evolved dramatically over the past decade. For the architects, developers and administrators of the software underpinning today's ubiquitous, mobile and context-aware IT systems, this evolution has brought significant new challenges. Key among these challenges is the need for software that adapts in a predictable way to continuously changing workloads, scenarios and user objectives.

Autonomic computing represents a promising approach to achieving such adaptiveness by adding self-management capabilities to the components of an IT system [15, 21, 22, 29]. Fig. 1 depicts the high-level architecture of an autonomic computing system. Given a set of user-specified policies (i.e., system objectives), an autonomic manager monitors the system components through sensors, uses its knowledge to analyse their state and to plan changes in their configurable parameters, and implements (or "executes") these changes through effectors.

We present a new framework comprising a software realisation of the architecture in Fig. 1, and a method for de-

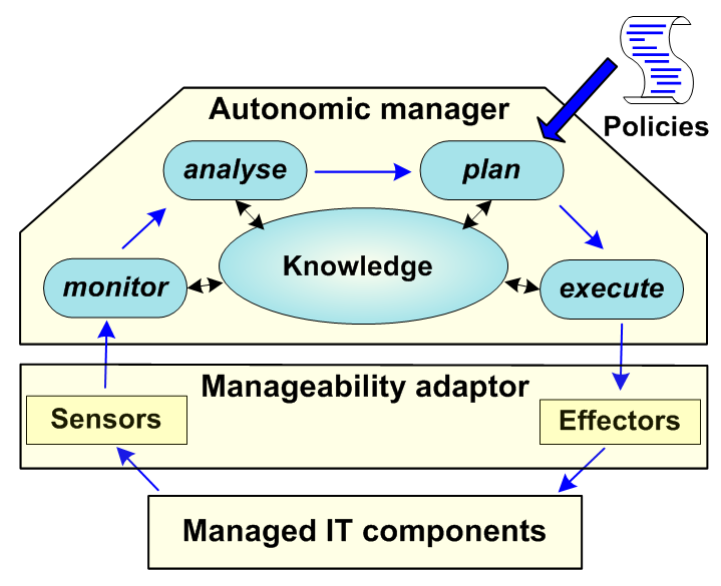

Figure 1. Autonomic computing system

veloping instances of this architecture for real-world IT systems. The novel characteristics of our framework are:

1) The knowledge within the autonomic manager consists of a continuous- or discrete-time Markov chain that models the behaviour of the managed IT components.

2) Runtime quantitative analysis of the Markov chain is employed for the analysis step in Fig. 1.

3) The autonomic manager is implemented as a web service that integrates the generic policy engine from [3] and the quantitative analysis tool PRISM [18].

4) Off-the-shelf tools are used for the computer-assisted generation of the manageability adaptor from Fig. 1 starting from the Markov chain.

5) The autonomic manager can be configured dynamically to manage any IT components whose behaviour can be specified by means of a Markov chain. To achieve this, a model of the IT components is generated from the Markov chain, and supplied to a running instance of the autonomic manager.

This framework has several significant advantages over existing approaches to autonomic system development. First, it is generic-our autonomic manager can be reconfigured for use with any legacy or future IT system whose behaviour (that is relevant to the planned autonomic application) can 


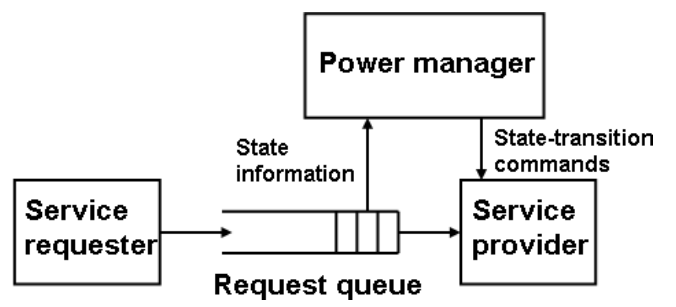

Figure 2. DPM-enabled device

be specified using a Markov chain. In contrast, current autonomic solutions use dedicated, proprietary autonomic managers for different applications (e.g., [4, 6, 19]). Second, the autonomic systems developed using the framework can implement a rich and flexible set of high-level policies that is unavailable in existing autonomic solutions. This is due to the broad spectrum of quantitative properties that can be specified in the temporal logics supported by PRISM $[16,17,18]$, the quantitative analysis tool integrated within our autonomic manager. Finally, the decisions taken by the autonomic manager are based on an exhaustive analysis of the user-specified policies and of the managed IT components. This powerful capability is made possible by our use of runtime quantitative analysis.

\section{Background}

Quantitative analysis of Markov chains PRISM [18] is a probabilistic model checker/quantitative analysis tool developed by the University of Oxford's Quantitative Analysis and Verification Group. The tool is used for the analysis of probabilistic models including discrete- and continuoustime Markov chains (DTMCs and CTMCs) [17] expressed in the PRISM high-level, state-based language.

Cost/reward-augmented versions of probabilistic computational tree logic (PCTL) [9] and continuous stochastic logic (CSL) [1] are used to specify the quantitative properties to analyse for DTMC and CTMC models, respectively [16]. To illustrate this process, we consider the dynamic power management (DPM) of a device with the architecture in Fig. 2. The device consists of a service provider that handles requests generated by a server requester and stored in a request queue. The service provider has several possible states corresponding to different power consumption and service rates, and its state transitions are controlled by a power manager that aims to optimise power consumption while maintaining an acceptable level of service for the device. Fig. 3 shows the PRISM representation of the CTMC model of a Fujitsu disk drive that matches the structure in Fig. 2, and which was introduced in [25].

The rewards... endrewards constructs in Fig. 3 define real values associated with certain CTMC states and transi-

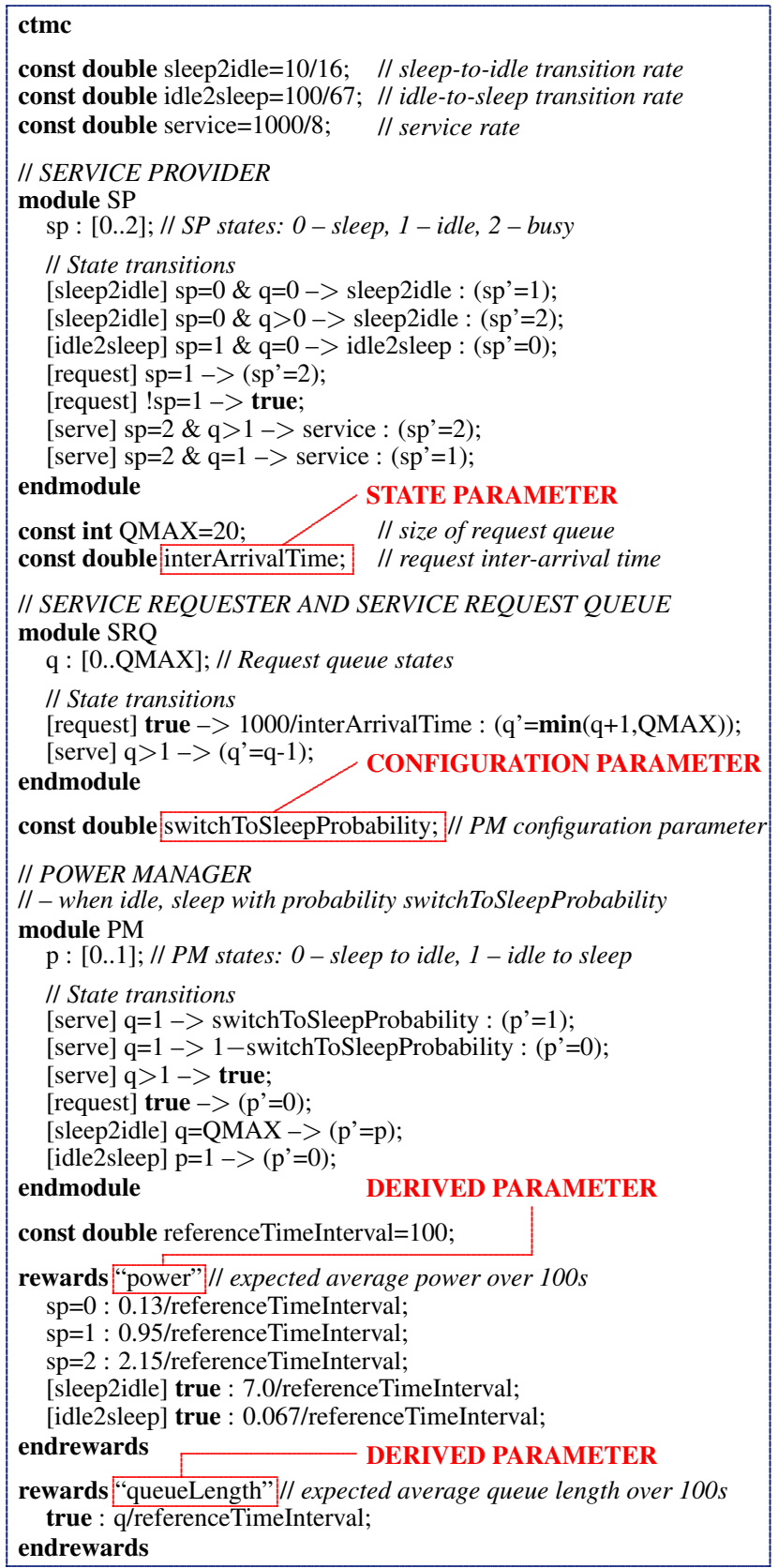

Figure 3. СTMC of a Fujitsu disk drive taken from [25]; a PRISM representation was available from [24].

tions. E.g., the "power" rewards construct associates:

- a value of $0.13 /$ referenceTimeInterval with the $s p=0$ (i.e., the sleep) state of the service provider

- and a value of $7.0 /$ referenceTimeInterval with its sleep2idle transition,

where 0.13 watts is the power consumed by the disk drive 


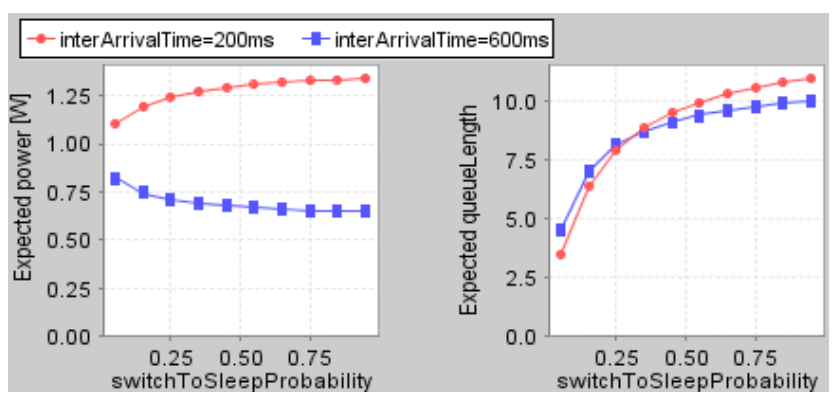

Figure 4. PRISM experiment: expected power usage and expected queue length for two request inter-arrival times and a range of "switch to sleep" probabilities.

in the sleep state, 7.0 joules is the energy used for a sleepto-idle transition, and referenceTimeInterval $>0$ represents the length of the time interval over which we intend to calculate the expected average power utilisation for the device. This calculation is performed by PRISM when presented with the "cumulative reward" property

$$
\mathrm{R}\{\text { "power" }\}=?[\mathrm{C} \leq \text { referenceTimeInterval }] .
$$

Similarly, the quantitative analysis of the PRISM property

$$
\mathrm{R}\{\text { "queueLength" }\}=?[\mathrm{C} \leq \text { referenceTimeInterval }]
$$

yields the expected average queue length during the first referenceTimeInterval seconds of operation. For a description of the techniques used for this analysis see [18].

The properties (1)-(2) can be evaluated for fixed values of the parameters interArrivalTime (i.e., the request interarrival time) and switchToSleepProbability (i.e., the probability for the service provider to be transitioned into the lowpower sleep state when it is idle). Alternatively, a PRISM experiment can be run to analyse these properties for a family of CTMCs whose members correspond to different values of the two parameters-Fig. 4. The autonomic manager introduced in this paper uses such PRISM experiments to analyse the current state of the managed IT components, and to plan changes in their configurable parameters that fulfil the user-specified policies for the system (cf. Fig. 1).

Our choice of PRISM for this role is due to its proven ability to analyse real-world systems that spawn a spectrum of application domains ranging from communication protocols and security systems to biological systems and system dependability $[17,18]$. An extensive, independent performance analysis of a range of probabilistic model checkers [13] ranked PRISM as the best tool for the quantitative analysis of large models such as the ones encountered in the autonomic systems targeted by our work.

Autonomic computing policies Several types of policies are used in autonomic systems [30, 31]:

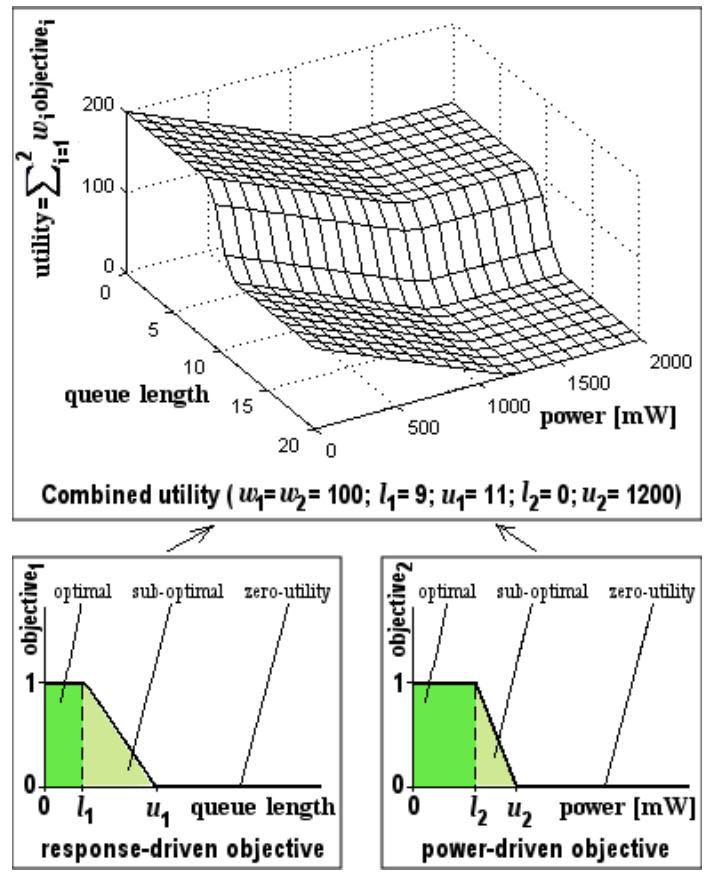

Figure 5. Utility function for the disk drive

- action policies provide a low-level specification of how the system configuration (i.e., the set of system parameters that can be modified by the autonomic manager) should be changed to match its state (i.e., the set of system parameters that can be read but not modified);

- goal policies specify precise constraints that the autonomic manager should meet;

- utility policies supply a "measure of success" that the autonomic system should optimise.

The use of runtime quantitative analysis within our framework enhances most the effectiveness of utility policies, which represent the most flexible of these policy types $[5,30]$. For this reason, the remainder of the paper will concentrate on our implementation of utility policies. Nevertheless, the policy engine used by the framework supports all policy types [3].

To illustrate the specification of utility policies, and without loss of generality, we will consider policies requiring the optimisation of multi-objective utility functions of the form:

$$
\text { utility }=\sum_{i=1}^{n} w_{i} \text { objective }_{i}
$$

where the weights $w_{i} \geq 0,1 \leq i \leq n$ are used to express the trade-off among the $n>0$ system objectives. Each objective function objective $_{i}, 1 \leq i \leq n$ is an analytical expression of the system state and configuration.

Fig. 5 depicts two such objective functions for the disk drive system described earlier in this section, and their combination into a multi-objective utility function. The analyti- 
cal form of the utility function is:

$$
\begin{aligned}
\text { utility }= & w_{1} \min \left(1, \max \left(0, \frac{u_{1}-\text { queueLength }}{u_{1}-l_{1}}\right)\right)+ \\
& w_{2} \min \left(1, \max \left(0, \frac{u_{2}-\text { power }}{u_{2}-l_{2}}\right)\right),
\end{aligned}
$$

where the lower bounds $l_{i}$ and the upper bounds $u_{i}$ from the two objective functions partition the value domains of queueLength (for $i=1$ ) and power (for $i=2$ ) into three regions: optimal - for values less than $l_{i}$; suboptimal-for values in $\left[l_{i}, u_{i}\right]$; and zero-utility-for values larger than $u_{i}$. This utility function can express a wide range of trade-offs between the power usage and the response time of the disk drive by adjusting the weights $w_{1}$ and $w_{2} \cdot{ }^{1}$

Policy engine The monitor-analyse-plan-execute loop within the autonomic manager in Fig. 1 is implemented by the generic policy engine that we introduced in [2, 3], and which we now extend to use runtime quantitative analysis for the implementation of utility policies.

As described in [3], the policy engine can manage IT components whose specification is supplied to the engine as part of a runtime configuration step. This specificationtermed a system model-defines the characteristics of every parameter of the IT components in the system, including its name, type (e.g., state or configuration) and value domain (e.g., integer, double or string). System models are represented as XML documents that are instances of a pre-defined meta-model encoded as an XML schema, and introduced in [2, 3]. This choice was motivated by the availability of numerous off-the-shelf tools for the manipulation of XML documents and XML schemasa characteristic largely lacking for the other technologies we considered. The policy engine is implemented as a .NET web service and takes advantage of object-oriented technology features such as polymorphism, reflection and generics in its handling of IT components whose characteristics are unknown until runtime.

\section{Generic Method for the Development of Autonomic IT Systems}

Our method for developing autonomic IT systems (Fig. 6) comprises three stages that correspond to the sets of steps to be performed by three different user roles: ${ }^{2}$

1) the manageability adaptor required to organise an existing IT system into an autonomic system is devised by the system developer during the generation stage;

2) in the deployment stage, this adaptor is deployed, and the policy engine is configured by the system administrator;

\footnotetext{
${ }^{1}$ The response time of the disk drive is provably proportional to the average length of the request queue [25].

${ }^{2}$ The same person may be responsible for two or all of these roles.
}

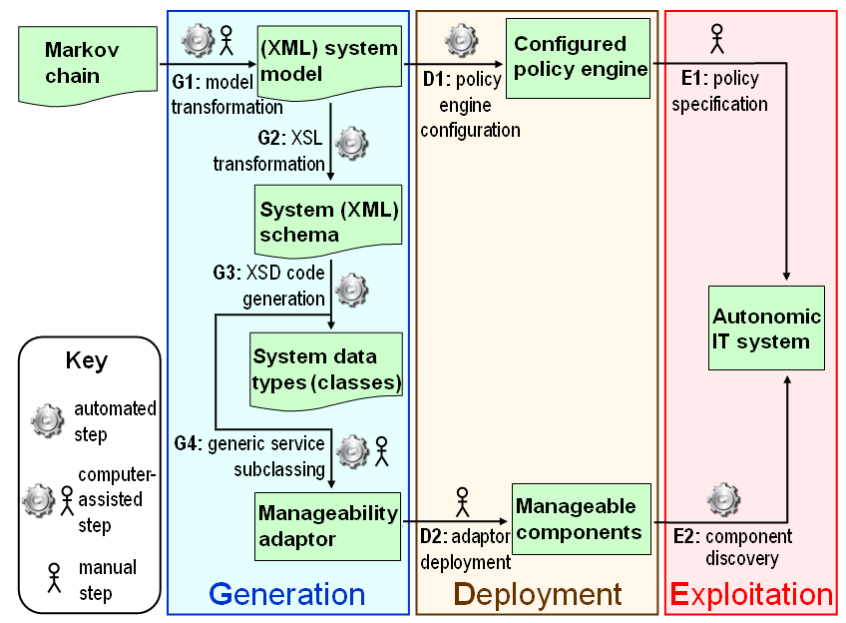

Figure 6. Autonomic system development

3) policies expressing the high-level system objectives are specified by the end user in the exploitation stage.

These stages are described below, and applied to the development of an autonomic DPM solution for the disk drive introduced in the previous section. The goal of the autonomic system developed in this running example is to ensure that the power manager from Fig. 2 adapts its decisions dynamically to changes in (a) the system workload; and (b) the user-specified utility policy for the DPM disk drive.

\subsection{Generation}

The first stage of the method starts from a PRISMencoded Markov chain describing the behaviour of the IT component(s) to be included in the autonomic system. This Markov chain may be available already from the development and/or verification of the IT component(s), or can be built as described in $[17,18]$. For our example, we use the CTMC of a Fujitsu disk drive shown in Fig. 3.

Step G1 In the first generation step, the Markov chain is used to derive an XML model for the configuration of the policy engine. The step is described by the mapping

$$
\text { modelTransformation : MarkovChain } \rightarrow \text { System, }
$$

where MarkovChain and System represent the set of Markov chains accepted by PRISM and the set of models used to configure the policy engine from [3], respectively. This requires the identification of the parameters of the IT components from the Markov chain by means of two rules:

1) Uninitialised const identifiers from the PRISM-encoded Markov chain (cf. Fig. 3) represent state or configuration parameters of the modelled IT component.

2) PRISM reward constructs represent parameters derived from the values of the other component parameters. 
A basic understanding of the semantics of the Markov chain is needed to distinguish between state and configuration parameters. Therefore, this transformation cannot be fully automated, but is performed in a computer-assisted fashion: the prototype model transformation tool that we implemented requires the system developer to specify the type of these parameters, and generates the other elements of the system model (including the value domain of each parameter) automatically.

As shown in Fig. 3, the disk drive from our running example has four parameters:

- interArrivalTime represents the double-valued request inter-arrival time, and is therefore a parameter associated with the system state.

- switchToSleepProbability $\in[0,1]$ is a configuration parameter that specifies the probability for the service provider to be transitioned into the low-power sleep state when it is idle.

- power and queueLength are two double-valued derived parameters.

In addition to these parameters, the generated model is augmented automatically with a string-valued parameter id that is used to distinguish between different instances of the modelled IT component, and a behaviouralModel parameter whose (string) value is the Markov chain itself.

Step G2 In this step, a standard XSLT engine (e.g., Saxon [27] in our prototype toolset) is used to apply a simple XSL transformation to the XML system model, and thus to automatically extract an XML schema specification for the targeted IT component(s):

$$
\text { schemaGen }: \text { System } \rightarrow \text { XmlSchema. }
$$

The result of this step for our running example is shown in Fig. 7. Note that for each derived component parameter with identifier $x$, a string-valued element $x$ Definition is automatically included in the XML complexType for the component (e.g., powerDefinition and queueLengthDefinition for the disk drive XML schema in Fig. 7). These elements are used to store the PRISM temporal logic properties that the policy engine will use to calculate the value of the derived component parameters at runtime.

Step G3 A standard data type generator (we use the XML Schema Definition tool [20]) is employed to generate the set of data types associated with the XML schema:

$$
\text { dataTypeGen : XmlSchema } \rightarrow \mathbb{P} \text { DataType. }
$$

This is a set of .NET classes in our framework.

Step G4 Finally, a simple transformation was implemented to automate the generation of manageability adaptor stubs for the components in the IT system:

adaptorGen $:$ XmlSchema $\rightarrow \mathbb{P}$ ManageabilityAdaptor.

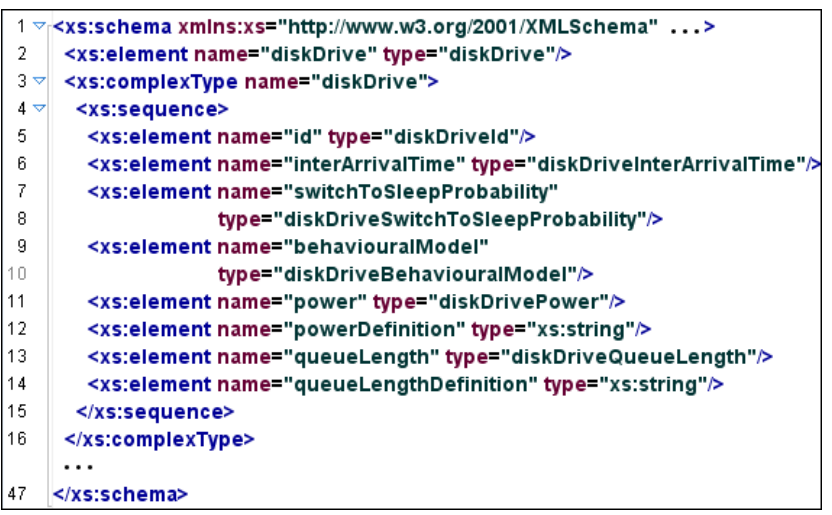

\section{Figure 7. Generated XML schema (Step G2)}
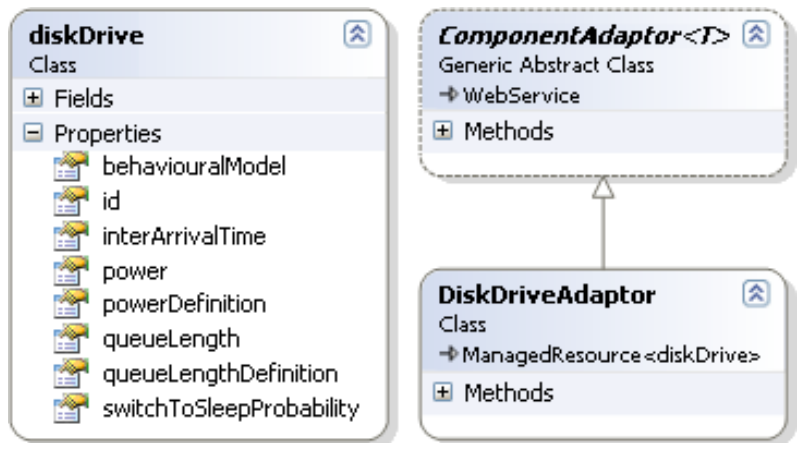

Figure 8. Class diagram for Steps G3-G4

As shown in Fig. 8 for our sample system, the manageability adaptors subclass a generic abstract web service ComponentAdaptor $\langle T\rangle$. This base class implements the bulk of the sensor and effector functionality associated with a manageability adaptor, hence only a small number of simple, component-specific methods that are declared abstract in this base class need to be implemented manually in these stubs as described in [3]. Implementing these methods for our system consisted in writing the code to get and set the disk-drive parameters from a discrete-event simulator that we developed to assess the effectiveness of the framework. Additionally, the powerDefinition and queueLengthDefinition fields of diskDrive objects were set to represent the PRISM properties (1)-(2).

\subsection{Deployment}

Step D1 In the first deployment step, the XML system model from Step G1 is supplied to the running instance of the policy engine that will be used in the autonomic system. Given the implementation of the engine as a web service, this involves the invocation of one of its web methods. To achieve this for our sample system, we uploaded the model using the web client implemented and described in [3].

When a system model is used to configure the policy en- 
gine, new data types and manageability adaptor proxies are generated within the engine to enable it to interoperate with the manageability adaptors for the component types specified in the system model. This fully automated code generation process is described in [3].

Step D2 The second development step consists of setting up the manageability adaptors built during the generation stage, and connecting them to the actual IT components to be included in the system. The first part of the operation represents a standard deployment of a web service, whereas the second part depends on the interface between the adaptor and the system components, but it typically involves configuring the two elements so that they know each other's address. For the system used in our running example, the URL of the discrete-event simulator was provided as a configuration parameter to the manageability adaptor.

\subsection{Exploitation}

Step E1 In this step, user-specified policies that express the objectives of the system as functions of its parameters are devised and supplied to the policy engine, e.g., by using the web client that was employed to upload the system model in Step D1. Typically, these policies are modified over time to reflect changes in the system goals. Depending on the configuration of the policy engine, the policies are evaluated and implemented either periodically or when the engine receives notifications about changes in the values of the system parameters from the manageability adaptors.

The policy handling techniques employed by the policy engine version that did not support quantitative analysis are described in [3]. In this section, we describe for the first time how the extended version of the policy engine is handling utility policies that rely on the use of runtime quantitative analysis. This will be done for the utility policy:

\section{MAXIMISE(scope, utility, configParam, min, max, step, mc)}

that uses the knowledge encoded in the Markov chain $m c$ to set the value of the configuration parameter configParam for each IT component within the scope of the policy. Runtime quantitative analysis is employed to examine all configParam values that are step units apart between min and max, and to select a value that maximises a multiobjective utility function with the form in (3).

The arguments in (9) are expressions that evaluate to a set of IT components (scope), a numerical value (utility, min, max and step) and the string-valued name of a component property (configParam and $m c$ ), respectively. For instance, the policy used for our running example is

$$
\begin{array}{r}
\text { Maximise('Type(.) = "diskDrive"', utility, } \\
\text { "switchTosleepProbability", }
\end{array}
$$

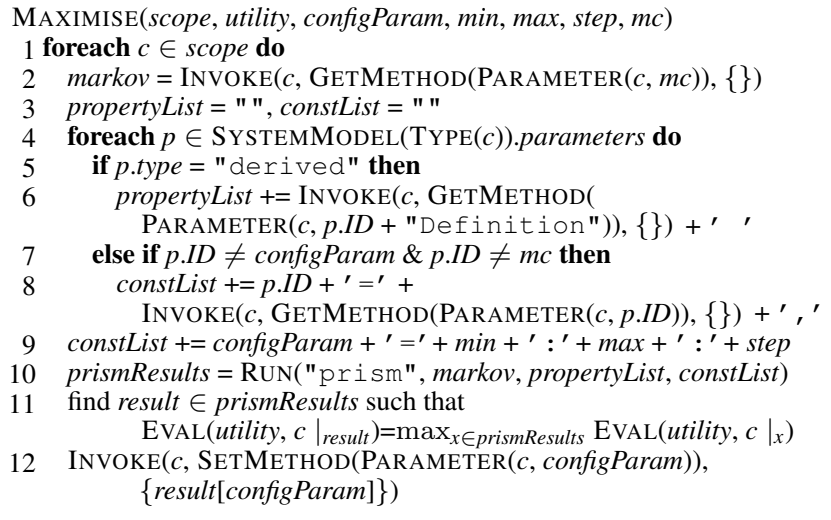

\section{Figure 9. Implementation of the utility pol- icy (9) using runtime quantitative analysis}

$$
\text { 0, 1,0.05, "behaviouralModel "), }
$$

where the utility function to optimise is given by (4).

Note that when the choice of step is such that the Markov chains corresponding to all possible configParam values are analysed, the optimal value will be identified and used at all times. When this is not feasible (e.g., for the switchToSleepProbability disk-drive parameter in (10)), the analysis may be impacted by local maxima. Offline quantitative analysis of the underlying Markov chain is then required to confirm the absence of local maxima and/or to choose the step parameter of policy (9) such that the resulting policy is effective. For our autonomic DPM system, the latter was made possible by the analysis available from $[24,25]$ and led to the choice of step $=0.05$.

The algorithm used to implement policy (9) within our policy engine is described by the pseudocode for the MAXIMISE function in Fig. 9. As policy (9) is local to each component in scope, ${ }^{3}$ the top-level foreach loop starting in line 1 implements the policy for each such component $c$ in turn.

First, lines 2-9 of the algorithm synthesise the three parameters required to run the command-line version of the PRISM tool for component $c$ : the Markov chain to analyse (markov); the quantitative properties to evaluate (propertyList); and the value ranges for the PRISM parameters in the Markov chain (constList). In line 2, reflection ${ }^{4}$ is used to obtain and invoke the Get method for the parameter of $c$ whose name is provided as the $m c$ argument of MAXIMISE. The result is stored as a string in markov. The foreach loop in lines 4-8 uses the TYPE operator to find out the type of $c$, looks up this component type in the SYSTEMMODEL supplied to the policy engine in the deployment step D1, and examines each of the component parameters. Component parameters of type 'derived' correspond

\footnotetext{
${ }^{3}$ A global policy is shown in Sect. 4.2.

${ }^{4}$ In object-oriented programming, reflection is a technique that enables programs to discover, create and manipulate classes and objects starting from their metadata [28].
} 
to reward constructs in the PRISM-encoded Markov chain (cf. Fig. 3), and the definitions of their associated temporallogic properties are concatenated into propertyList (lines 56 ). The values of all other component parameters (except configParam and $m c$ ) are used to synthesise the commaseparated list of PRISM parameter values constList in lines 7-8. The PRISM encoding of the configParam values to be analysed (i.e., "configParam=min:max:step") is then appended to the resulting constList in line 9.

The PRISM experiment is run in line 10, and the results of this runtime quantitative analysis are parsed into an $\left(\left\lfloor\frac{\text { max-min }}{\text { step }}\right\rfloor+1\right)$-element array prismResults. For each analysed configParam value $x_{i}=\min +i \cdot$ step, $0 \leq$ $i \leq\left\lfloor\frac{\text { max }- \text { min }}{\text { step }}\right\rfloor$, prismResult $[i]$ is a dictionary that maps configParam to $x_{i}$, and the name of each 'derived' parameter of $c$ (e.g., "power" and "queueLength" for a diskDrive component) to the value that the parameter would take if configParam $=x_{i}$.

In line 11 , the utility function is evaluated for the considered values of configParam. For each dictionary $x \in$ prismResult, the parameters of $c$ that $x$ maps to values are set to these values, and utility is calculated for the resulting object $\left.c\right|_{x}$ using an auxiliary function EvaL. One of the prismResult elements that maximise utility is chosen as the analysis result, and used in line 12 to set the value of the configParam field of $c$. On exit from MAXimise, the new configParam values for the IT components in scope will be enforced using the appropriate manageability adaptors, as described in [3].

The outermost loop starting in line 1 is performed \#scope times. Lines 2-3 take constant time, and so does the foreach loop in lines $4-8$ because an IT component has a constant number of parameters to examine and handle. Line 9 takes constant time, while running PRISM to analyse a temporal logic property takes $t_{P R I S M}>0$ time units. A detailed analysis of $t_{P R I S M}$ is available in [17]; here, we will just state that $t_{P R I S M}$ is linear in the size of the analysed property, and quadratic in the state size of the analysed Markov chain. Evaluating utility for a single configParam value in line 11 takes constant time, hence choosing a value that maximises utility will take $\mathrm{O}\left(\frac{\max -\min }{\text { step }}\right)$ time. Finally, setting the value of configParam in line 12 takes constant time. The overall complexity of the algorithm is therefore $\mathrm{O}\left(\#\right.$ scope $\left.\left(t_{\text {PRISM }}+\frac{\text { max-min }}{\text { step }}\right)\right)$-linear in the number of IT components to which autonomic capabilities are added, in the time taken to analyse the Markov chain using PRISM, and in the number of analysed configParam values.

Step E2 The policy engine detects automatically all manageable IT components that have been registered with its component discovery service, and whose types are specified in the system model used for its configuration.

\section{Case Studies}

\subsection{Dynamic Power Management}

We start our presentation of case studies with the experimental results for the autonomic DPM system from Sect. 3. A discrete-event simulator was implemented to evaluate policy (10) for a simulated disk drive handling requests with exponentially distributed inter-arrival time. Each experiment was for a one-hour period in simulated time. The mean request inter-arrival time was varied in the range $200 \mathrm{~ms}$ to $2000 \mathrm{~ms}$ in each experiment, and the parameters $w_{i}, l_{i}, u_{i}, 1 \leq i \leq 2$ from (4) were varied across experiments. This allowed us to assess the ability of the system to adapt to changes in its workload and objectives, respectively. Additionally, two standard DPM methods were also applied to each of the experimental data sets in order to contrast the autonomic DPM method with existing DPM techniques. The two standard DPM methods selected were ([25]): the timeout method, which moves the disk drive into the sleep state after a period of idleness $t$ and "awakens" it immediately after a request has arrived; and the $N$ method, which moves the disk drive into the sleep state as soon as it becomes idle, and "awakens" it after $N$ requests accumulate in its queue.

The experimental results are summarised in Fig. 10. The graphs contrast the average actual utility achieved by our autonomic disk drive with the average actual utilities achieved by the timestamp and $\mathrm{N}$ methods. For the timestamp method, different experiments were run for each considered disk drive workload, and for each $500 \mathrm{~ms}$-apart value of $t$ in $[0,10 \mathrm{~s}]$. The results shown in Fig. 10 correspond to the choice of $t$ yielding the highest average actual utility, so that the autonomic method is compared with the best possible instance of the timestamp method. Likewise, N-method experiments were run for all $0 \leq N \leq 10$, and the best result is represented in the graphs. The actual utilities are shown as a percentage of the maximum value of the utility function (4) (i.e., $w_{1}+w_{2}$ ), for multiple values of the $w_{1} / w_{2}$ ratio between the weights of the power-related and performancerelated terms of the utility function. The chosen $w_{1} / w_{2}$ ratios cover a broad range of power/performance trade-offs, (i.e, $w_{1} / w_{2} \in\{0.1,0.2, \ldots, 1.0,2.0, \ldots, 10.0\}$ ), and are represented on the horizontal axes using logarithmic scale.

Each graph corresponds to a different combination of the $l_{i}, u_{i}$ parameters of the utility function (4), $1 \leq i \leq 2$. For the top row of three graphs, the performance-related utility parameters are fixed at relatively demanding values (i.e., $l_{1}=8$ and $\left.u_{1}=9\right)$, and the power-related requirements are relaxed from left to right (cf. Eq. (4) and Fig. 5). The graphs in the bottom row correspond to much more relaxed performance requirements (i.e., $l_{1}=18$ and $u_{1}=20$ ), and to the same power-related parameters $l_{2}, u_{2}$ as the top row. 

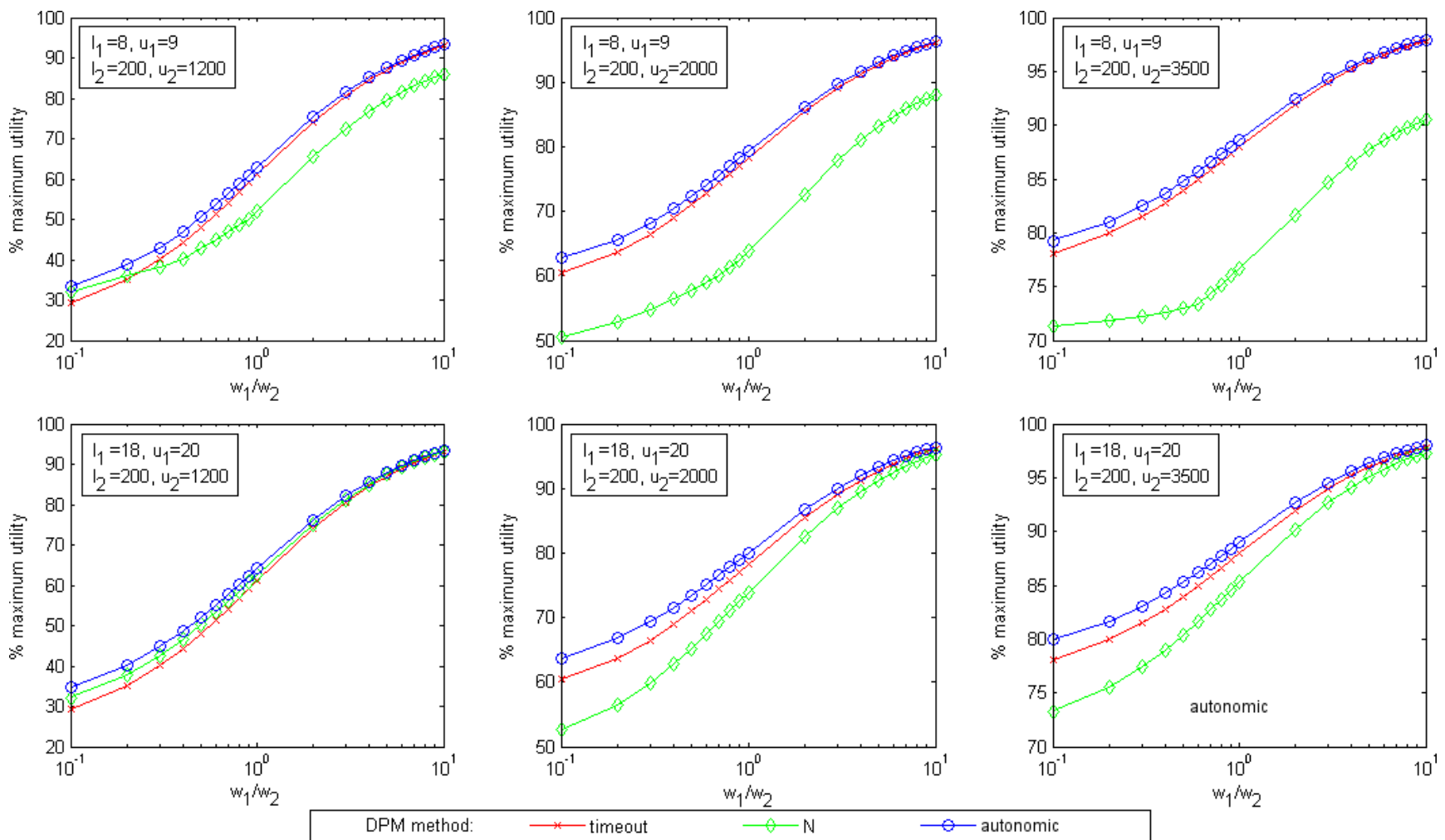

Figure 10. Experimental results for the DPM disk drive

Note that a straightforward way to enable users with limited IT expertise to select an effective policy instance for their needs is to present them with a graphical user interface (GUI) comprising a "power/performance trade-off" slider GUI control that adjusts the $w_{1} / w_{2}$ ratio, and a radio-button GUI control for selecting a family of policy instances such as the ones corresponding to the six graphs in Fig. 10.

The experimental results show that the autonomic disk drive achieves better utility than the timeout and $\mathrm{N}$ methods across a broad range of performance/power trade-offs. For all experiments, the autonomic DPM method betters the other methods by a wider margin for small to medium values of $w_{1} / w_{2}$, i.e., when the power-related term of (4) has a major contribution to the overall utility. In these areas of the graphs, the autonomic DPM method adjusts the configuration of the disk drive to its workload in ways that are not accessible to the timeout and $\mathrm{N}$ heuristics for any considered value of their parameters $t$ and $N$, respectively.

For large values of $w_{1} / w_{2}$, the three DPM methods achieve similar actual utility. The explanation is that for these $w_{1} / w_{2}$ ratios the power-related term of the utility function (4) is dwarfed by the performance-related term, and both standard methods can maximise the performancerelated term for certain values of their parameters. These values are $t=10 \mathrm{~s}$ for the timeout method (i.e., the disk drive is almost never moved into the sleep state), and $N=0$ for the $\mathrm{N}$ method (i.e., an idle disk drive wakes up as soon as a request enters its queue). Hence, DPM is of limited use when the utility function is nearly independent of the power usage; in this case, the utility value can be optimised by simply maintaining the disk drive active at all times.

The policy evaluations were done at regular, 10 -second time intervals. For our simple CTMC, the execution of the algorithm in Fig. 9 took sub-second time on a $3 \mathrm{GHz}$ dualcore AMD-processor desktop running Windows XP. The average CPU overhead was in the range 1.5\%-2.5\%, and techniques for decreasing it are discussed in Sect. 6.

As a final remark, note that when applied to hardware IT components such as disk drives, our method provides functionality similar to that of automatic control applications. What distinguishes our method from these applications is the ability to reconfigure the policy engine at its core for use across application domains, and its model-driven approach to IT component management using autonomic policies to express the high-level system objectives.

\subsection{Cluster Availability Management}

The second case study involves the adaptive control of cluster availability within a data centre. The objective of this application is to control the number of servers allocated to the $N \geq 1$ clusters of a data centre in order to maximise 


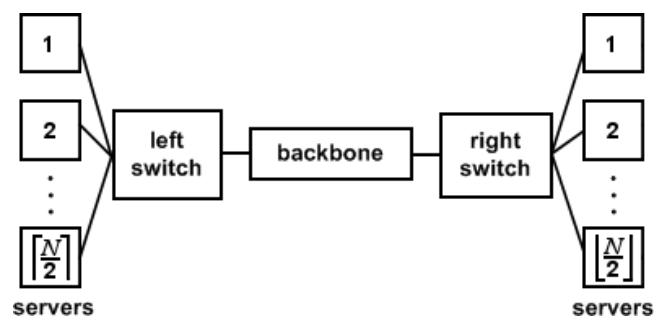

Figure 11. $N$-server dependable cluster [10]

the utility function

$$
\text { utility }=\sum_{i=1}^{N} w_{i} \cdot \operatorname{GOAL}\left(\text { availability }_{i} \geq l_{i}\right)-w_{0} \sum_{i=1}^{N} \text { server }_{i}
$$

subject to

$$
\sum_{i=1}^{N} \text { servers }_{i} \leq \text { Total_servers and required } i \leq \text { servers }_{i},
$$

where $w_{i}>0$, availability $_{i} \in[0,1], l_{i} \in[0,1]$,required $_{i} \geq$ 1 and servers $_{i} \geq 1$ represent the priority, expected availability, target availability, number of required servers, and number of (allocated) servers for cluster $i, 1 \leq i \leq N$, respectively. The parameters $w_{i}$ and $l_{i}$ have the same role as in (4), but we used the operator GOAL( availability $_{i} \geq l_{i}$ ) instead of $\min \left(1, \max \left(0, \frac{u_{i}-\text { availability }_{j}}{u_{i}-l_{i}}\right)\right)$ like in the objective functions from (4) in order to express the degenerate case when $u_{i}=l_{i}$. The GOAL operator yields 1 when its argument is true and 0 otherwise, Total_servers $\geq 1$ is the total number of servers in the data centre, and $0<$ $w_{0} \ll 1$ is a constant. ${ }^{5}$ The expected availability of cluster $i$, availability ${ }_{i}$, was defined as the expected fraction of a one-year time period during which at least required ${ }_{i}$ servers are usable (i.e., they are operational, connected to an operational switch, etc.), assuming that the cluster configuration does not change.

As the utility function (11) is defined across all clusters within a data centre, the associated policy is a global policy. Its implementation is achieved using a variant of the algorithm in Fig. 9 that combines the per-component results of the runtime quantitative analysis instead of deciding the value of $\operatorname{servers}_{i}, 1 \leq i \leq N$ on a per cluster basis.

Like in the previous case study, we applied the method introduced in Sect. 3 starting from an existing Markov chain representation of the targeted IT component, i.e., the CTMC of a dependable cluster of workstations introduced in [10]. This CTMC takes into account the failure and repair rates of all components from our targeted cluster architectureshown in Fig. 11. Consequently, the policy engine can use

\footnotetext{
${ }^{5}$ The second term of (11) ensures that when multiple configurations maximise the first sum, the configuration using the fewest servers is preferred.
}

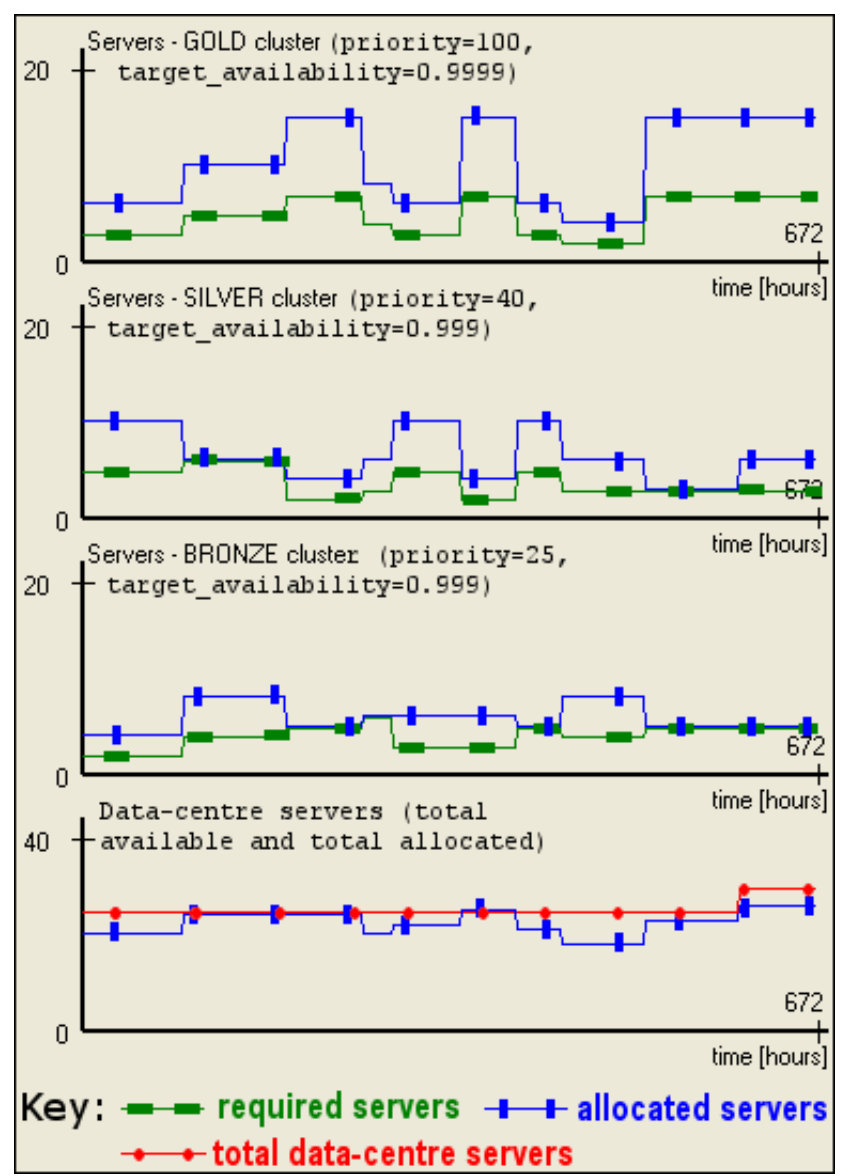

\section{Figure 12. Simulation results for a three- cluster data centre over a four-week period}

PRISM experiments to calculate the expected cluster availabilities for the data-centre configurations that satisfy (12), and to decide the number of servers that each cluster should get so that the value of the utility function (11) is maximised. Given the complexity of the CTMC behavioural model, we configured the policy engine to employ the notification mechanism of the cluster manageability adaptor, and recalculate the server allocations only when the system state changed. With all possible data-centre configurations analysed by the PRISM experiments, this calculation took up to 30 seconds with the policy engine running on the same desktop PC as before. This response time is acceptable for our use case because, based on our previous experience with policy-based data centre management [4], half a minute represents a small delay compared to the time required to provision a server when it is allocated to a new cluster. ${ }^{6}$

The step parameter of policy (9) was set to 1 , so that all possible values of the configuration parameter servers $s_{i}$ were considered during the runtime quantitative analysis, and the

\footnotetext{
${ }^{6}$ Sect. 6 suggests techniques for working around the time taken by runtime quantitative analysis when such delays are not acceptable.
} 
chosen configuration was guaranteed to be optimal. For this reason, we present only a set of typical experimental results for this case study (Fig. 12) rather than contrasting these results with those corresponding to other availability management techniques. Maximising the three-cluster data centre utility involves allocating sufficient servers to achieve the target availability of the highest priority cluster GOLD at all times. During time intervals when all three clusters require large numbers of servers, this impacts the ability of the lower priority clusters SILVER and BRONZE to realise their target availabilities, and only the required numbers of servers are allocated to these clusters. The second term of the utility function (11) ensures that servers are not allocated to clusters unless this can improve the system utility. Keeping more servers unassigned in this way is advantageous, as the overhead to provision a spare server for inclusion into a cluster is significantly lower than the overhead to move it from one cluster to another.

\section{Related Work}

A number of other projects have addressed the modelbased development of autonomic systems. In [14], the authors define an autonomic architecture meta-model that extends IBM's autonomic computing blueprint [12], and use a model-driven process to partly automate the generation of instances of this meta-model. Each instance is a specialpurpose organic computing system that can handle the use cases defined by the model employed for its generation. Our framework eliminates the need for the 19-activity generation process described in [14] by using a generic policy engine that can be dynamically reconfigured to handle any use cases in which the behaviour of the system components can be modelled by means of a Markov chain.

Several research projects propose the use of ModelDriven Architecture (MDA) techniques to develop autonomic computing policies and autonomic systems starting from high-level behavioural models of the system or of its components [7, 23, 26]. Two of these approaches [7, 23] are targeted at bespoke systems whose components already exhibit sophisticated autonomic behaviour, and therefore cannot be readily extended to handle generic legacy components. In contrast, our generic framework can accommodate any type of IT component for which a probabilistic model is available. The preliminary work described in [26] is closer to our approach in that it advocates the importance of using MDA techniques in the development of generic autonomic systems; however, the authors do not substantiate their proposal with any concrete solution, but rather qualify it as an open challenge. Our framework represents an important first step towards addressing this challenge.

In [11], the authors take a view similar to ours by introducing a paradigm termed model-driven autonomic com- puting, and explaining that the model-based validation of self-management decisions represents a more reliable and flexible approach than the use of pre-set policies. A hierarchical model of NASA's Autonomous Nano-Technology Swarm missions is successfully used in [11] to achieve the self-managing functionality that these missions depend on, and thus to illustrate the benefits of the approach. Our work complements the results in [11] with a new model-based approach to developing autonomic functionality, and proposes a generic method that uses existing tools and standards for the implementation of autonomic systems.

\section{Conclusion}

Boosted by the development of powerful quantitative analysis tools $[13,16]$, the use of probabilistic model checking for the static analysis of the quantitative properties of real-world systems has become increasingly popular in recent years [17]. In this paper, we extended the use of Markov chains and quantitative analysis to the development and runtime operation of autonomic IT systems.

Starting from a PRISM-encoded Markov chain available from the verification of a legacy IT system or built as described in $[17,18]$, the generic method for the development of autonomic functionality introduced in the paper uses automated and computer-assisted techniques to devise a selfmanaging version of the original system. The three stages of the method can be carried out by a system developer with experience in quantitative analysis using PRISM (the generation stage); by a system administrator with minimal experience in web service deployment (deployment); and by a technically competent user (exploitation).

The paper describes for the first time an extended version of the policy engine from [3] that uses runtime quantitative analysis to implement powerful user-specified policies that would have been extremely complicated to express and support otherwise. The runtime use of quantitative analysis involves the automated synthesis of the parameters for the execution of a PRISM experiment [18], including the generation of appropriate values and value sets for the undefined constants in the PRISM Markov chain, and the assembly of the PRISM probabilistic temporal logic properties to be evaluated. The results of the quantitative analysis are then automatically interpreted and used to select an appropriate new configuration for the system, ensuring that it continually adapts to changes in its environment and in the userspecified policies.

Runtime quantitative analysis can incur significant overheads in terms of both response time and resource (e.g., CPU and memory) utilisation. The use of a subscriptionnotification mechanism as described in Sect. 4 is one way to reduce these overheads by avoiding the unnecessary periodical re-evaluation of policies. Several other options that 
we are planning to investigate include: the use of caching and pre-evaluation techniques to bypass the analysis step during policy evaluation; performing the quantitative analysis within the process space of the policy engine; and the use of a hybrid approach in which a less demanding PRISM experiment is carried out to produce a close-to-optimal configuration for the autonomic system and a faster technique is then used to refine this configuration.

Other areas of future work are the development of additional applications to assess the effectiveness of our framework within new application domains; and the design of policies supporting an extended set of probabilistic system properties, along the lines of the results reported in [8]. Finally, a major challenge for the future is the development of online machine learning techniques for the synthesis and/or refinement of a Markov-chain model for an IT system, e.g., starting from an approximate behavioural model provided by its administrator.

Acknowledgement This work was partly supported by the UK Engineering and Physical Sciences Research Council grant EP/F001096/1.

\section{References}

[1] A. Aziz et al. Model checking continuous time Markov chains. ACM Transactions on Computational Logic, 1(1):162-170, 2000.

[2] R. Calinescu. Model-driven autonomic architecture. In Proc. 4th IEEE Intl. Conf. Autonomic Computing, 2007.

[3] R. Calinescu. Implementation of a generic autonomic framework. In D. Greenwood et al., editor, Proc. 4th Intl. Conf. Autonomic and Autonomous Systems, pages 124-129, 2008.

[4] R. Calinescu and J. Hill. System providing methodology for policy-based resource allocation, July 2004. United States Patent Application no. 10/710322.

[5] R. Das et al. Towards commercialization of utility-based resource allocation. In Proc. 3rd IEEE Intl. Conf. Autonomic Computing, pages 287-290, 2006.

[6] S. Ghanbari et al. Adaptive learning of metric correlations for temperature-aware database provisioning. In Proc. 4 th IEEE Intl. Conf. Autonomic Computing, June 2007.

[7] D. Gracanin et al. Towards a model-driven architecture for autonomic systems. In Proc. 11th IEEE Intl. Conf. Engineering of Computer-Based Systems, pages 500-505, 2004.

[8] L. Grunske. Specification patterns for probabilistic quality properties. In Proc. 30th Intl. Conf. Software Engineering, pages $31-40,2008$.

[9] H. Hansson and B. Jonsson. A logic for reasoning about time and reliability. Formal Aspects Comp., 6(5):512-535, 1994.

[10] B. Haverkort et al. On the use of model checking techniques for dependability evaluation. In Proc. 19th IEEE Symp. Reliable Distributed Systems, pages 228-237, October 2000.

[11] M. Hinchey et al. Modeling for NASA autonomous nanotechnology swarm missions and model-driven autonomic computing. In Proc. 21st Intl. Conf. Advanced Networking and Applications, pages 250-257, 2007.
[12] IBM Corporation. An architectural blueprint for autonomic computing, 2004. http://www03.ibm.com/autonomic/pdfs/ACBP2_2004-10-04.pdf.

[13] D. Jansen et al. How fast and fat is your probabilistic model checker? An experimental comparison. In K. Yorav, editor, Hardware and Software: Verification and Testing, volume 4489 of LNCS, pages 69-85. Springer, 2008.

[14] H. Kasinger and B. Bauer. Towards a model-driven software engineering methodology for organic computing systems. In Proc. 4th Intl. Conf. Computational Intelligence, pages 141146, July 2005.

[15] J. Kephart and D. Chess. The vision of autonomic computing. IEEE Computer Journal, 36(1):41-50, January 2003.

[16] M. Kwiatkowska. Quantitative verification: Models, techniques and tools. In Proc. 6th Joint Meeting of the European Software Engineering Conf. and the ACM SIGSOFT Symp. Foundations of Software Engineering, pages 449-458. ACM Press, September 2007.

[17] M. Kwiatkowska et al. Stochastic model checking. In M. Bernardo and J. Hillston, editors, Formal Methods for the Design of Computer, Communication and Software Systems: Performance Evaluation, pages 220-270. Springer, 2007.

[18] M. Kwiatkowska et al. Quantitative analysis with the probabilistic model checker PRISM. Electronic Notes in Theoretical Computer Science, 153(2):5-31, 2005.

[19] C. Lefurgy et al. Server-level power control. In Proc. 4th IEEE Intl. Conf. Autonomic Computing, June 2007.

[20] Microsoft Corporation. XML schema definition tool, 2007. msdn2.microsoft.com/en-us/library/x6c1kb0s(VS.80).aspx.

[21] R. Murch. Autonomic Computing. IBM Press, 2004.

[22] M. Parashar and S. Hariri. Autonomic Computing: Concepts, Infrastructure \& Applications. CRC Press, 2006.

[23] J. Pena et al. A model-driven architecture approach for modeling, specifying and deploying policies in autonomous and autonomic systems. In Proc. 2nd IEEE Intl. Symp. Dependable, Autonomic and Secure Computing, pages 19-30, 2006.

[24] PRISM Case Studies: Dynamic Power Management. http://www.prismmodelchecker.org/casestudies/power.php.

[25] Q. Qiu et al. Stochastic modeling of a power-managed system: construction and optimization. In Proc. Intl. Symp. Low Power Electronics Design, pp. 194-199. ACM Press, 1999.

[26] M. Rohr et al. Model-driven development of self-managing software systems. In Proc. 9th Intl. Conf. Model-Driven Engineering Languages and Systems. Springer, 2006.

[27] SAXON - The XSLT and XQuery Processor. http://saxon.sourceforge.net/.

[28] J. Sobel and D. Friedman. An introduction to reflectionoriented programming. In Proc. Reflection'96. 1996.

[29] R. Sterritt and M. Hinchey. Biologically-inspired concepts for self-management of complexity. In Proc. 11th IEEE Intl. Conf. Eng. Complex Computer Systems, pages 163168, 2006.

[30] W. Walsh et al. Utility functions in autonomic systems. In Proc. 1st Intl. Conf. Autonomic Computing, pp. 70-77, 2004.

[31] S. White et al. An architectural approach to autonomic computing. In Proc. 1st IEEE Intl. Conf. Autonomic Computing, pages 2-9. IEEE Computer Society, 2004. 